\title{
Epileptic EEG signal classifications based on DT-CWT and SVM classifier
}

\author{
S.Deivasigamani*, G.Narmadha** C.Senthilpari***, Wong Hin Yong ${ }^{* * *}$ and P.K.Rajesh**** \\ * Faculty of Engineering and Computer Technology, AIMST University, Malaysia. \\ ** Department of Electrical and Electronics Engineering, Sethu Institute of Technology, Tamilnadu, India. \\ *** Faculty of Engineering, Multimedia University, Malaysia. \\ **** Faculty of Medicine, AIMST University, Malaysia. \\ *Corresponding Author: deivasigamani@aimst.edu.my
}

Submitted : 10/05/2020

Revised :04/02/2021

Accepted :22/02/2021

\begin{abstract}
Contamination in human cerebrum causes the mind issue, which is epilepsy. The contaminated territory in the cerebrum area creates the unpredictable example signals as focal signs, and the other sound locales in the mind produce the standard example signals as nonfocal sign. Henceforth, the discovery of focal signs from the nonfocal signs is significant for epileptic medical procedure in epilepsy patients. This paper proposes a straightforward and proficient technique for Electroencephalogram (EEG) signals orders utilizing Support Vector Machine (SVM) classifier. The exhibition of the proposed EEG signals characterization framework is assessed for Sensitivity, Specificity, and Accuracy.
\end{abstract}

Keywords: Epilepsy; Focal; SVM; Neural networks; Epileptogenic area.

\section{INTRODUCTION}

The headache, stroke, and Alzheimer's diseases are the notable issues in the human brain. In this arrangement of clutters, epilepsy is another sort of cerebrum issue, which haphazardly happens in huge populace nations. Epilepsy is a typical neurological issue influencing youngsters and grown-ups. Early determination and treatment have been related with diminishing bleakness and mortality. This is particularly if the kind of epilepsy has been affirmed, and proper administration has been started. EEG keeps on being the highest quality level recognition strategy. The signs, which are procured by EEG sensor, are nondirect and complex in its tendency. Consequently, it is extremely convoluted cycle to distinguish and separate the moment changes in acquired EEG signals (Karthik et al. 2020).

The cathodes are set on the scalp of the human head, and EEG signals are caught through various channels. The signs that are caught from the epileptogenic territory are called focal EEG signals, and the signs that are caught from the other piece of the epileptogenic region are called non-ocal EEG signals. In this way, it gets essential for an automatic recognition and characterization framework to be proposed for the focal and non-focal EEG signs to continue with epileptic medical procedure and further treatment. Epileptic seizures create abnormal functionalities in the brain region, and they are captured from the brain, and the identification of focal and nonfocal 
signs from EEG signals is important for epileptic surgery. In developing nations, with an immense populace, it gets fundamental for a PC helped automatic detection of location and classification procedure for the epileptic seizures to be arranged from the EEG signals. The focal and nonfocal EEG signals are ordered naturally in this examination work to restrict the epileptic seizure for epileptic medical procedure. The fundamental inspiration of this examination work is to make mindfulness about the epilepsy malady in the individuals and give a decent and proficient answer for the early distinguishing proof of Epilepsy illness in patients. In the present Epilepsy identification strategy, the patient experiences the brain waves screening by specialists in wellbeing focuses, and they will caution the patient in the event that they are influenced by Epilepsy issue.

This paper proposes a computer supported programmed location of epilepsy issue through the examination of EEG signals. The area discovery of epileptogenic territory in the cerebrum is significant for prior epilepsy medical procedure. Epileptogenic region is the segment of the brain, where the synapses are seriously influenced by infection, and this influencing territory of the cerebrum produces the signs that are known as focal signs, as shown in Fig. 1 (a). The nonfocal signs are created by the sound tissue regions in the mind, as shown in Fig. 1 (b). This paper proposes a computer aided automatic characterization of focal and nonfocal signs utilizing Support Vector Machine (SVM) classifier. The characterizations pace of these regular order algorithms such as Random Forest (RF), Deep Learning Neural Network (DLNN), and Discrete Wavelet Transform (DWT) is low, and it is not appropriate for additional seriousness conclusion measure (Durga Devi et al. 2020).

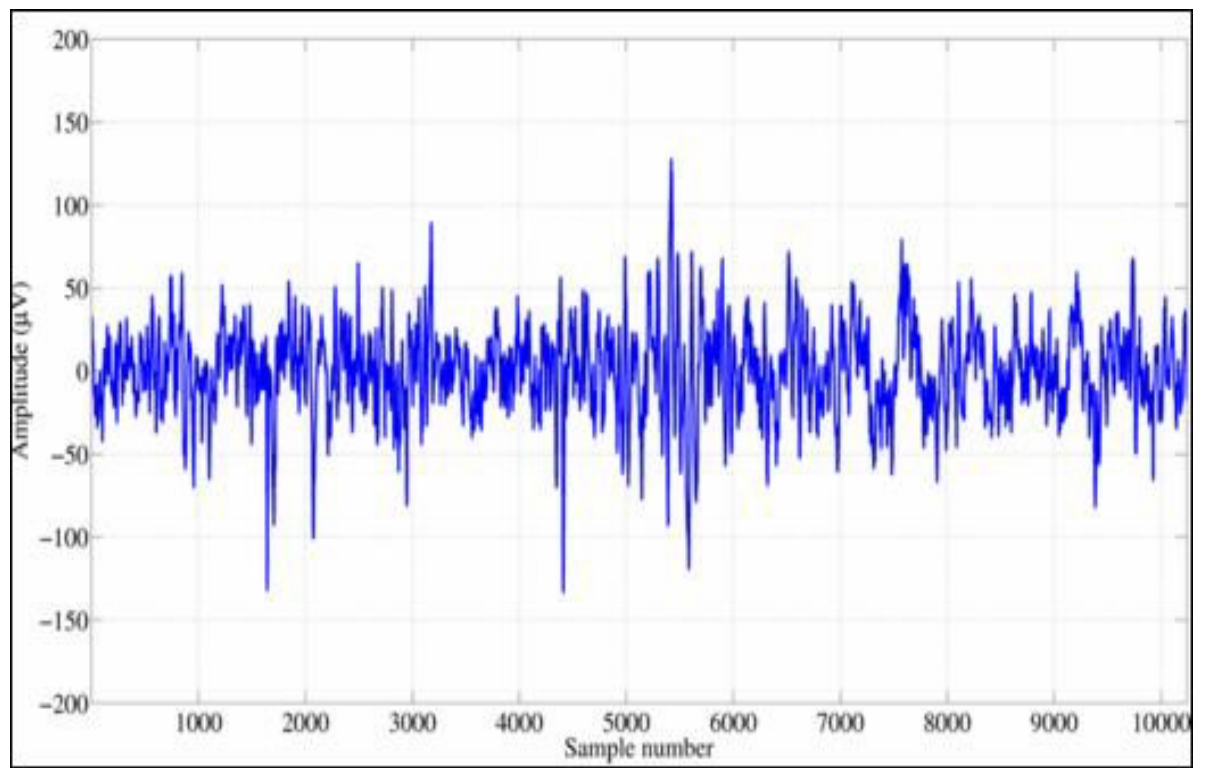

(a) 


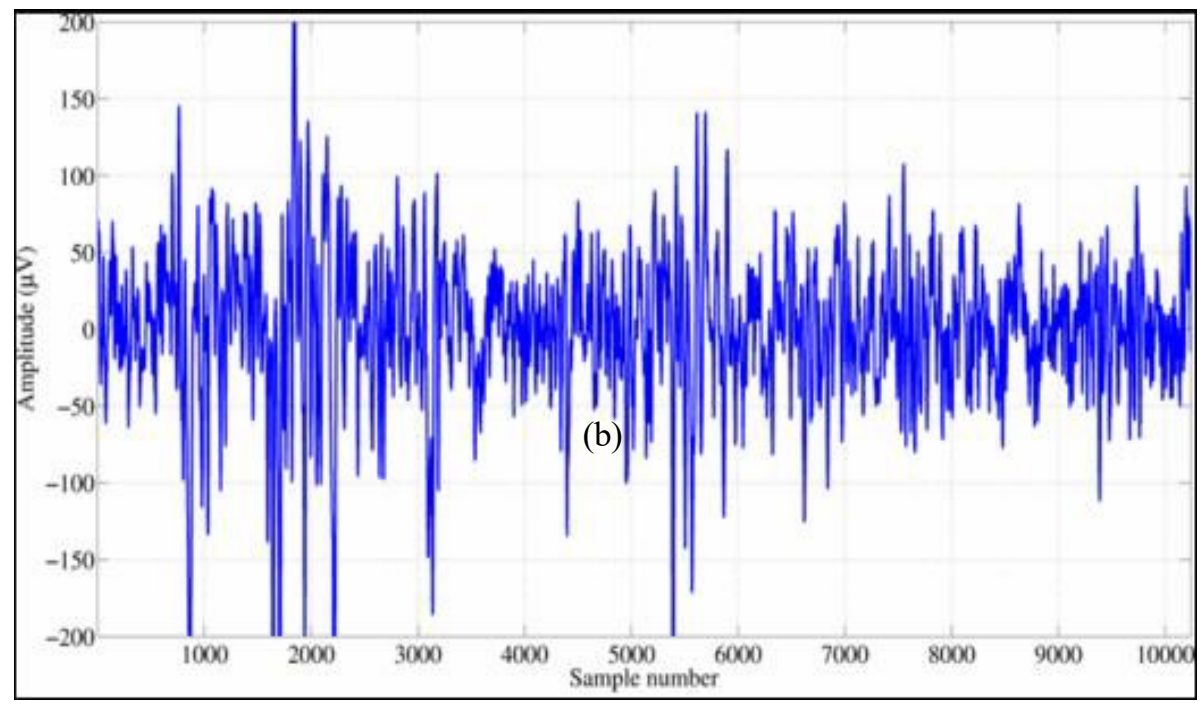

Figure 1. (a) Focal signals. (b) Nonfocal signals.

This paper is composed of 5 segments. Section 2 discusses the different traditional strategies for the automatic classification of EEG signals for epilepsy issue recognition. Section 3 proposes a basic and productive approach to distinguish and characterize the EEG signals utilizing SVM classifier. Section 4 discusses the trial details, and lastly, Section 5 delineates the conclusion.

\section{RELATED WORKS}

Akbarian and Erfanian et al. (2019) survey the programmed seizure identification dependent on nonlinear dynamical investigation of EEG signals and common data. Research on epilepsy was progressively being done on open access information, of EEG designs accessible in a store. Pisano et al. (2019) utilized self-sorting out maps for developing nonexponential polynomial calculation. These self-sorted-out maps from this polynomial procedure were created and investigated by different arrangement of nonstraight classification rules. The EEG signals utilized in this paper were quantized at the inspecting rate $50 \mathrm{~Hz}$, and they were disintegrated with the guide of Daubechies wavelet family mode.

Wavelet transform is recorded similar to a successful device to distinguish EEG seizures (Yuan et al, 2018). This was utilized for profound learning methods. In this article, the writers examine how the constraints of profound learning procedures can be limited by embracing a multi setting combination approach. Acharya et al. (2018) grew profound convolutional neural system based automatic location and distinguishing proof of EEG signals for Epilepsy malady. Bonn University dataset was utilized in this paper to assess the proposed Convolutional Neural Network (CNN) based epilepsy malady recognition technique. The normal sensitivity record rate was about $90 \%$, the normal estimation of the specificity list rate was about $88.7 \%$, and the normal estimation of accuracy file rate was about $95 \%$. Wei Zhao et al. (2020) achieved 93.55\% accuracy with a five-class classification method using one dimensional CNN.

Dodge et al. (2017) grew profound convolutional neural system based automatic recognition and distinguishing proof of EEG signals for Epilepsy infection. The normal sensitivity record rate was about 92.6\%, the normal estimation of the specificity file rate was about $93.8 \%$, and the normal estimation of accuracy file rate was about 
96.8\%. Pushpendra Singh et al. (2017) applied Fourier change for the order procedure of focal signs from nonfocal EEG signals for the recognition of Epilepsy infection with utilizing SVM characterization. The authors accomplished $94.1 \%$ of a normal accuracy for the signs classifications.

AnindyaBijoy Das et al. (2016) arranged EEG signals for investigation the unusual occasions related with cerebrum area. The observational mode area technique was utilized for signals classifications. The authors accomplished $89.4 \%$ of a normal accuracy and $90.7 \%$ of a normal sensitivity. Sharma et al. (2016) applied time situated frequency confined orthogonal wavelet channel banks for the location of epileptic seizure EEG signals from the nonepileptic seizure EEG signals utilizing least mean square SVM order. This proposed technique for EEG signal grouping framework accomplished $91.95 \%$ of sensitivity rate, $96.56 \%$ of specificity rate, and

$94.25 \%$ of EEG signal order exactness rate utilizing the proposed strategy.

Kumar et al. (2015) utilized Gabor transformation filter on the source EEG signals for acquiring the changed filter signal. This changed sifted signal was applied to the component extraction module, which was included by separating technique arriving at $88.33 \%$ of EEG signal characterization accuracy. Sharma et al. (2015) utilized least square sort SVM classifier, which arranged the EEG signals into focal and nonfocal signs with a normal accuracy rate of $87 \%$.

Table 1. Analysis the survey on classification methodology for EEG signals classifications.

\begin{tabular}{|c|c|c|}
\hline Authors & Methodologies & Limitations \\
\hline Pushpendra Singh et al. (2017) & SVM & Low classification accuracy \\
\hline Taqi et al. (2017) & $\begin{array}{c}\text { Convolutional soft-max } \\
\text { classification algorithm }\end{array}$ & Detection delay is high \\
\hline Akshata Patted et al. (2016) & NN classifier & Not reliable \\
\hline Das et al. (2016) & KNN classifier & Low sensitivity rate \\
\hline Ahammad et al. (2014) & Linear classifier & Detection delay is high \\
\hline Yuan et al. (2011) & Neural networks and SVM & Loss in frequency pattern occurs \\
\hline Abibullaev et al. (2010) & Basis wavelet function & Low classification accuracy \\
\hline Minasyan et al. (2010) & $\begin{array}{c}\text { Recurrent neural network } \\
\text { classification model }\end{array}$ & . \\
\hline
\end{tabular}

\section{PROPOSED METHODOLOGY}

The EEG signals are caught from the cerebrum by putting 19 terminals over the skull of the brain. Dual tree complex wavelet transform is applied on these caught EEG signals, and the spatial area signals are changed over into recurrence space signals for additional classifications. The features are extracted from the transformed wavelet coefficients, and afterward, the SVM classifier is utilized to characterize the EEG signals into either focal or nonfocal depending on the separated list of features. The proposed system for EEG signals classification is delineated in Fig. 2 . 


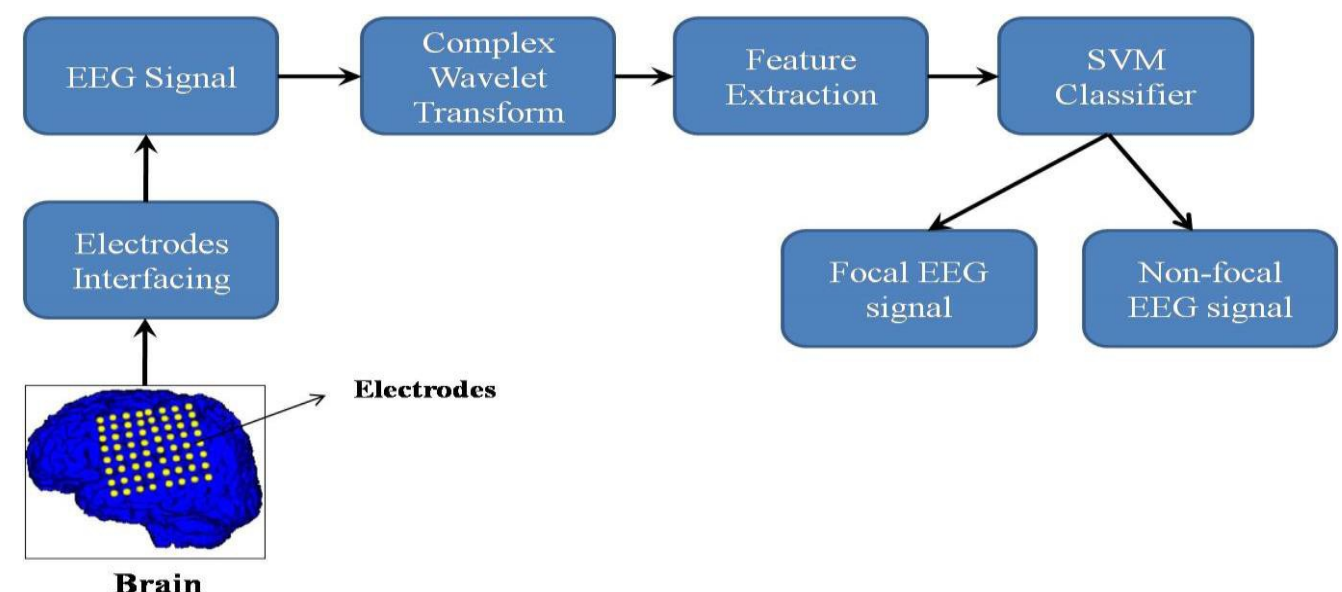

Figure 2. Proposed methodologies for EEG signals classifications.

The EEG recordings utilized in this paper to test the order execution of epileptic seizures are gathered from the open database accessible at the University of Bern-Barcelona EEG dataset (2012). The dataset included EEG accounts of patients who have been experiencing long haul intracranial medicines at the Department of Neurology, University of Bern, Switzerland. These EEG signals were tested at a pace of $512 \mathrm{~Hz}$ or $1024 \mathrm{~Hz}$ dependent on the quantity of channels, for example, more than 64. The database incorporates 750 focal EEG signals and 750 nonfocal EEG signals gotten from contiguous channels. In this work, 100 focal and 100 nonfocal sets of EEG signals are haphazardly looked over in this dataset for programmed EEG signal arrangements.

\section{Dual Tree Complex Wavelet Transform}

In this paper, dual tree complex wavelet transform (DT-CWT) is utilized so as to change over the spatial space signal into recurrence area signal, as expressed in Yuan et al. (2011). The DT-CWT is applied on the test sign, and it produces lower order and higher-order coefficients at two continuous stages. Propositions coefficients show the direct connection among lower and higher order changed signs. The deterioration stage comprises low pass channel, which creates the low pass channel coefficients; what is more, there is high pass channel, which delivers high pass channel coefficients. The procedure of disintegration continues until it arrives at the required phase of decomposition. In this paper, 4 stages of decomposition were used.

\section{Feature Extraction}

Features are the significant job in EEG signal arrangements. Features separate the focal signs from nonfocal signs utilizing their attributes. The accompanying highlights are extracted from the coefficients of complex wavelet transform.

Contrast $=\sum\left(|i-j|^{2} \times p(i, j)\right)$ 
where ' $n$ ' represents the number of subbands in the DT-CWT. 'Di,j' represents the extracted coefficients from EEG signal.

Energy $=\sum_{i=1}^{n}\left|D_{i, j}\right|^{2} ; j=1, \ldots n$

Entropy determines the signal energy per unit slot in EEG signal, where ' $p$ ' represents the number of signals, and $\mathrm{P}(\mathrm{i}, \mathrm{j})$ represents the extracted coefficients of complex wavelet transform.

Entropy $=-\sum p(i, j)\left[\log _{2} p(i, j)\right]$

In equations 1 and $4, p(i, j)$ represents the coefficients of complex wavelet transform and ' $\mathrm{i}$ ' and ' $\mathrm{j}$ ' represent its corresponding coordinates. ' $\mu$ ' depicts the mean and ' $\sigma$ ' represents its variance.

\section{Classifier}

SVM is the most often administered classifier and precise learning system. It yields effective grouping and brings about different application spaces, for instance, clinical determination, content arrangement, face acknowledgment, and bioinformatics. SVM depends on the basic hazard minimization rule from the measurable learning hypothesis. The part controls the observational hazard and arrangement limit so as to boost the margin between the classes and limit the expenses as stated in Anuja et al. (2020). The engineering of SVM

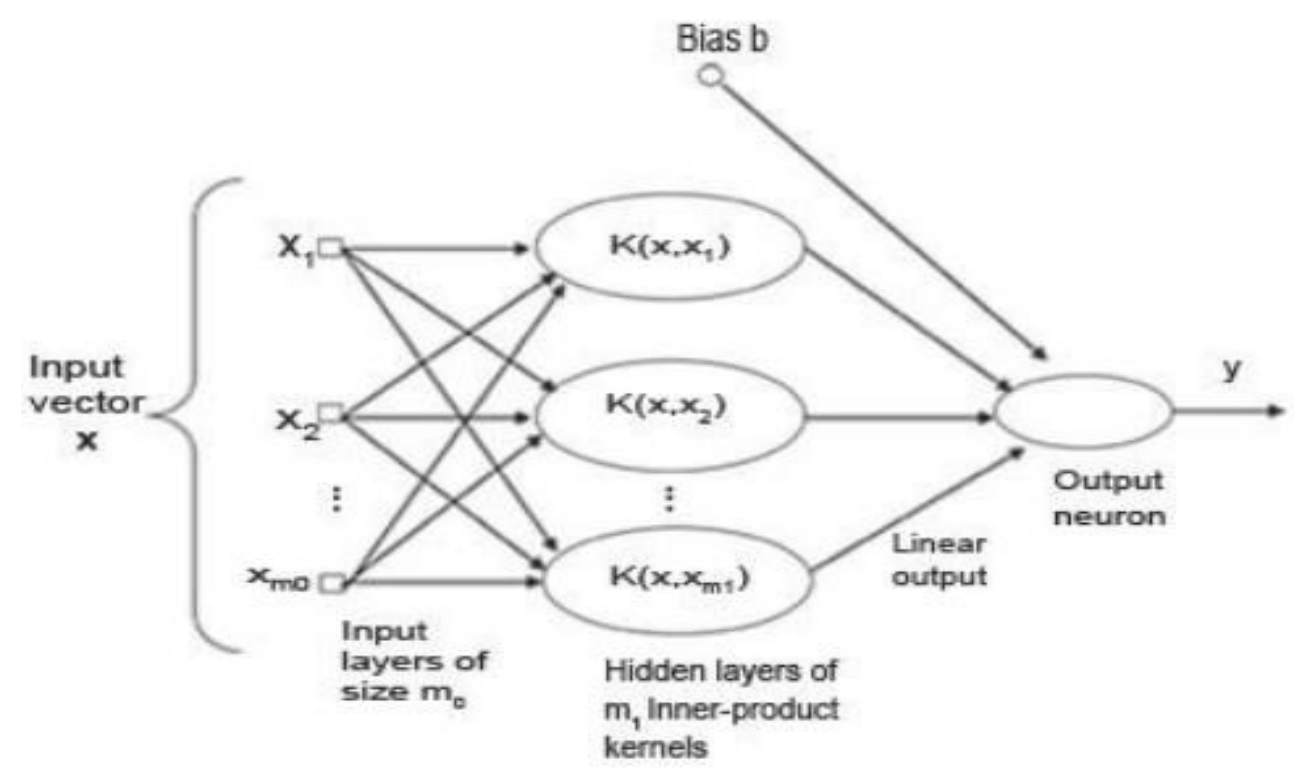

Figure 3. Architecture of SVM Classifier. 
The objective in preparing a SVM is to discover the isolating hyper plane with the biggest margin. It is to discover the weight ' $w$ ' that augments the margin ' $b$ '. It comprises an information layer, shrouded layer and yield layer. The SVM is utilized to make an indicator work from a lot of preparing information where the capacity itself can be a twofold, a multi-classification, or even a general relapse indicator. The SVM finds a hyper surface which endeavors to part the positive and negative models with the biggest conceivable margin on all sides of the hyper plane. On account of direct detachable information, the straight SVM attempts to discover among all hyper planes that limit the preparation mistake, the one that isolates the preparation information with greatest good ways from their nearest focuses (maximal margin hyper plane). This hyper plane for paired example characterization issues is given in equation 4 . The upside of straight classifiers is that have straightforward preparing calculations that scale well with the quantity of models.

$\mathrm{y}_{\mathrm{i}}\left[\mathrm{w}^{\mathrm{T}} \mathrm{x}_{\mathrm{i}}+\mathrm{b}\right] \geq 1, \ldots, \mathrm{n}$

where $\mathrm{x}_{\mathrm{i}}$ is the preparation information having a place with either class $\mathrm{y}_{\mathrm{i}}=[-1,+1]$, $\mathrm{w}$ is weight parameter and $\mathrm{b}$ is a predisposition parameter. The maximal margin hyperplane is the one that fulfils the compels of equation 4 , while simultaneously limits $\|w\|^{2}$. At that point, a quadratic streamlining issue with disparity compels is settled utilizing the Lagrangian technique by amplifying equation 5 subject to equation 6 .

$L_{d}(a)=\sum_{i=1}^{n} a_{i}-\frac{1}{2} \sum_{i, j-1}^{n} y_{i} y_{j} a_{i} a_{j} x_{i}^{T} x_{j}$

$\mathrm{a}_{\mathrm{i}} \geq 0 ; \sum_{\mathrm{i}=1}^{\mathrm{n}} \mathrm{a}_{\mathrm{i}} \mathrm{y}_{\mathrm{i}}=0$

SVM will in general perform better when applied to information outside the preparation set when contrasted with different kinds of classifiers. The SVM accomplishes great execution by concentrating on the preparation models that are progressively hard to characterize, the alleged support vectors.

In this paper, SVM classifier is used to detect and differentiate the focal signals from nonfocal signals. SVM classifier has two distinct modes as training and testing. During the training mode of the classifier, the extracted features from focal and non-focal signals are trained individually and the trained patterns are stored for signal classifications. During the testing mode of the classifier, the extracted features from the test EEG signals are classified with respect to the trained patterns which are obtained from the training mode of the classifier.

The testing mode of the SVM classifier produces binary output, in which ' 0 ' represents the focal signal and ' 1 ' represents the non-focal signal.

\section{RESULTS AND DISCUSSIONS}

Bern-Barcelona EEG dataset is used in this paper to validate the proposed EEG signal classification system. This dataset contains 750 focal and 750 nonfocal EEG signals, captured from various patients in Bern University Hospital. These captured EEG signals in both cases are sampled at the rate of $512 \mathrm{~Hz}$. In this paper, we have used 50 focal signals and 50 nonfocal signals. The performance of the proposed EEG signal classification system is analyzed in terms of sensitivity, specificity, and accuracy. These parameters are given as 
Sensitivity $(\mathrm{Se})=\frac{T P}{(T P+F N)}$

Specificity $(S p)=\frac{T N}{(T N+F P)}$

$\operatorname{Accuracy}(A c c)=\frac{T P+T N}{(T P+F P+T N+F N)}$

True positive is noted as TP, and it defines the number of signals correctly classified as focal signals. True negative is noted as TN, and it defines the number of signals wrongly classified as focal signals. False positive is noted as FP, and it defines the number of correctly classified nonfocal signals, and false negative is noted as FN, and it defines the number of wrongly classified nonfocal signals. The performance of the proposed system is illustrated in Table 2.

Table 2. Performance analysis.

\begin{tabular}{|c|c|}
\hline $\begin{array}{c}\text { Performance evaluation } \\
\text { parameters }\end{array}$ & $\begin{array}{c}\text { Results achieved } \\
(\mathbf{\%})\end{array}$ \\
\hline Sensitivity (Se) & 97 \\
\hline Specificity (Sp) & 98 \\
\hline Accuracy (Acc) & 96 \\
\hline
\end{tabular}

Table 3 shows the performance comparisons of the proposed classification methodology with conventional methodologies for focal and nonfocal signal classifications. The proposed methodology achieves $97 \%$ sensitivity, $98 \%$ specificity, and $96 \%$ average accuracy for focal and nonfocal signals classifications, as illustrated in Table 2.

Table 3. Performance comparisons with existing methodology.

\begin{tabular}{|c|c|c|c|}
\hline Methodology & Year & $\begin{array}{c}\text { Signals in } \\
\text { Dataset }\end{array}$ & Accuracy (\%) \\
\hline Proposed & 2020 & 100 & 96 \\
\hline Wei Zhao et al. & 2020 & 100 & 93.55 \\
\hline Pisano et al. & 2019 & 9 & 89.75 \\
\hline Acharya et al. & 2018 & 100 & 95 \\
\hline Pushpendra Singh et al. & 2017 & 50 & 94.1 \\
\hline
\end{tabular}




\begin{tabular}{|c|c|c|c|}
\hline AnindyaBijoy Das & 2016 & 100 & 89.4 \\
\hline Sharma et al. & 2015 & 50 & 87 \\
\hline Sharma et al. & 2014 & 50 & 85 \\
\hline Zhu et al. & 2013 & 50 & 84 \\
\hline
\end{tabular}

\section{CONCLUSION}

In this paper, computer aided automatic classification of focal and nonfocal EEG signals is proposed for epilepsy disorder diagnosis. The dual tree complex wavelet transform is applied, and then features are extracted from these transformed coefficients. Then, SVM classifier classifies the EEG signals into either focal or nonfocal based on the trained features. The proposed system stated in this paper achieves $97 \%$ sensitivity, $98 \%$ specificity, and $96 \%$ average accuracy for focal and nonfocal signals classifications. For diagnosis purposes, detection accuracy is important. The DT-CWT and SVM method for the detection system of focal EEG signal have high accuracy than the existing methodology. In future, this proposed work can be extended for the detection of stroke in brain through the diagnosed focal EEG signals.

\section{REFERENCES}

Akbarian, B., \& Erfanian, A. (2019). Automatic Seizure Detection Based on Nonlinear Dynamical Analysis of EEG Signals and Mutual Information. Basic and Clinical Neuroscience, 9(4), 227-240. http://dx.doi.org/10.32598/bcn.9.4.227:

Pisano, B., Teixeira, C.A., Dourado, A., and Fanni, A. (2019). Application of Self Organizing Map to Identify Nocturnal Epileptic Seizures. Neural Computing and Applications, 10.

Ye Yuan, Guangxu Xun, KebinJia \& Aidong Zhang (2018). A multi-context learning approach for EEG epileptic seizure detection. BMC Systems Biology, 12 (Suppl 6), 107.

Acharya, U.R., Oh, S.L., Hagiwara, Y., Tan, J.H., and Adeli, H. (2018). Deep convolutional neural network for the automated detection and diagnosis of seizure using EEG signals. Computers in Biology and Medicine, 100, 270-278.

Dodge, S., Karam, L. (2017). A study and comparison of human and deep learning recognition performance under visual distortions. In: 26th IEEE International Conference on Computer Communication and Networks (ICCCN), 1-7.

Pushpendra Singh, Ram BilasPachori. (2017). Classification of focal and non-focal EEG signals using features derived from fourier-based rhythms. Journal of Mechanics in Medicine and Biology, 17.

Das, A.B. and Bhuiyan, M.I.H. (2016). Discrimination and classification of focal and non- focal EEG signals using entropy-based features in the EMD-DWT domain. Biomedical Signal Processing and Control, 29(2016), 11-21.

Bhattacharyya, A., Sharma, M., Pachori, R.B., Sircar, P., Acharya, U.R. (2016). A novel approach for automated detection of focal EEG signals using empirical wavelet transform. Neural Computing and Applications, 1-11. 
Kumar, T. S. Kanhangad, V. and Pachori, R. B. (2015). Classification of seizure and seizure-free EEG signals using local binary patterns. Biomedical Signal Processing and Control, 15, 33-40.

Sharma, R., Pachori, R.B., Acharya, U.R. (2015). An integrated index for the identification of focal electroencephalogram signals using discrete wavelet transform and entropy measures. Entropy, 17(8), 52185240 .

Taqi, A.M., Al-Azzo, F., Mariofanna, M., Al-Saadi, J.M. (2017). Classification and discrimination of focal and non-focal EEG signals based on deep neural network. Proc International Conference on Current Research in Computer Science and Information Technology (ICCIT), Slemani, 86-92.

Patted, A, Bekal, S. and Desai, V. (2016). EEG Signal Classification into Seizure and Non- Seizure Class using Discrete Wavelet Transform and Artificial Neural Network. International Journal of Innovative Research in Computer and Communication Engineering, 4, 1-7.

Ahammad, N. Fathima, T. and Joseph, P. (2014). Detection of epileptic seizure event and onset using EEG. BioMed Research International, 2014, 1-7.

Yuan, Q., Zhou, W., Li, S. and Cai, D. (2011). Epileptic EEG classification based on extreme learning machine and nonlinear features. Epilepsy Res, 96, 29-38.

Abibullaev, B. Kim, M.S. and Seo, H.D. (2010). Seizure detection in temporal lobe Epileptic EEGs using the best basis Wavelet functions. Journal of Medical Systems, 34, 755-765.

Minasyan, G.R. Chatten, J.B. and Harner, R.N. (2010). Patient-specific early seizure detection from scalp EEG. Journal of Clinical Neurophysiology, 27, 163-178.

Sharma, R., Pachori, R.B., and Gautam, S. (2014). Empirical mode decomposition based classification of focal and non-focal EEG signals. Proc Int Conf Medical Biometrics, 135-140.

Zhu, G. Li, Y. Wen, P. P. Wang, S. Xi, M. (2013). Epileptogenic focus detection in intracranial EEG based on delay permutation entropy. Proc AIP Conference, 1559, 31-36.

Karthik, B., Krishna Kumar, T., Vijayaragavan, S.P(2020), "Removal of high density salt and pepper noise in color image through modified cascaded filter", J Ambient Intell Human Comput, pp. 1-8,

Durga Devi, T.J.B., Subramani, A. \& Anitha, (2020) "Modified adaptive neuro fuzzy inference system based load balancing for virtual machine with security in cloud computing environment", J Ambient Intell Human Comput, , pp. 1-8.

Wei Zhao, Wenbing Zhao, Wenfeng Wang, Xiaolu Jiang, Xiaodong Zhang, Yonghong Peng, Baocan Zhang, Guokai Zhang, (2020) "A Novel Deep Neural Network for Robust Detection of Seizures Using EEG Signals", Computational and Mathematical Methods in Medicine, vol. 2020, Article ID 9689821, 9 pages, https://doi.org/10.1155/2020/9689821.

Anuja S B, Usha Nandini K, Sukanya S.T, (2020) "ECG Signals Classification using Statistical and Wavelet Features", International Journal of Recent Technology and Engineering (IJRTE), Volume-8 Issue-5, January 2020, pp.1497-1504. 